\title{
The Comparative Effect of Hydro Alcoholic and Hydro Distillation Extracts of Melissa officinalis on Acne and Pimple
}

\author{
Giti Sabet Teymouri ${ }^{1, a}$, Mozhgan Sabet Teimouri ${ }^{2, b^{*}}$ \\ ${ }^{1}$ Researcher-Manager of Tebyan Toos Institute, Mashhad, Iran, ${ }^{2}$ Academic Centre and Education \\ for Culture and Research, Khorasan Razavi Organization, Mashhad, Iran \\ ${ }^{b *}$ ecotijdm@gmail.com
}

Keywords: Acne; hydro alcoholıc extracts; hydro dıstıllatıon extracts; melissa; pımple

\begin{abstract}
One of the famous plants in family Lamiaceae is Melissa officinalis which is one of the important herbs for health and Medicine. Melisa contains Rosmarinic acid, flavonoids and phenolic. These components have tightening and rejuvenation effect on skin. Also, it has monoterpenes and di-terpenes which are antibacterial. Essential oil of Melisa forms a good complex with sebum, so it can decrease inflammation and acne pain $30 \mathrm{gr}$ of dry leaves and stems of Melisa in $500 \mathrm{ml}$ of distilled water were used for hydro-distillation. For hydroalcoholic extract, we used $150 \mathrm{gr}$ of dry matter (leaves and stems) in $500 \mathrm{ml}$ of ethyl alcohol at $25^{\circ} \mathrm{C}$ for 10 days. Then, the alcoholic extract was diluted 5 times with water 20 persons were under experiment for 3 months. Extracts were sprayed on skin 3-5 times a day. The results showed that all the people treated got the best result of this treatment. After the application of the water extract of Melissa, we observed $75 \%$ of clients improved the skin pore, $65 \%$ of clients' improved neurological problems and Acne infections and $92 \%$ recovery of hypersecretion of Sebaceous glands. We observed the same results with Hydro Alcoholic extracts. So that $75 \%$ recovery of clients with improved skin pore, $95 \%$ of clients with improved neurological problems and Acne infections and $90 \%$ recovery of hypersecretion of Sebaceous glands. The results showed that Melisa extract has value of applying on some skin problems.
\end{abstract}

\section{Introduction}

In the last decade, people's interest in taking medicinal herbs has increased due to familiarity with the adverse effects of chemical drugs, so that herbal medicines are used to treat most diseases.

Complementary medicine refers to the use of complementary and alternative medicine along with conventional medicine. The use of medicinal plants is one of the treatments used in complementary and alternative medicine [1]. Traditional herbal remedies are used alone or in combination with other methods to treat acne in Europe.

Among these plants, Melissa officinalis L. is one of the most well-known medicinal plants in Lamiaceae family. Lemon balm (Melissa) is an aromatic perennial herb that belongs to them [2, 3]. Melissa has long been used in traditional medicine to treat many disorders, and several studies have been conducted to identify the plant and its healing properties. Major producing countries are Italy $(5000 \mathrm{~kg})$, France $(1500 \mathrm{~kg})$, Hungary $(1500 \mathrm{~kg})$, Iran $(700 \mathrm{~kg})$, Argentina $(500 \mathrm{~kg})$ Yugoslavia $(500 \mathrm{~kg})$ and Israel $(200 \mathrm{~kg})[4,5]$.

Genuine Melissa oil is pale yellow sweet smelling oil with an odour of aromatic lemon. Yields are as low as $0.014 \%$ for distillation of the fresh herb to $0.112 \%$ for dried herb, increasing to $0.13 \%$ using cohobation. True oil is extremely expensive. The oil is held in high esteem for its use in aromatherapy, it is reported to have a sedative effect [6]. The result of some study showed that the Wild Melissa growth in some part of the Golestan forest, Gonbad-e-Kavuos, Azerbaijan, Kermanshah, Lorestan, Tehran, Qazvin province of Iran and planted in many farms there.

Also this herb contains volatile oil $(0.2-0.8 \%)$ which the most important compounds. Some important components in Melissa essential oil are Geranium, Nerium, Citronellal, Linalool, Geranyl acetate, Methyl Citronellate, Caryophyllene oxide, Germacrene, Eugeno. In some research, scientist introduced some another compounds same that Rosemarinic acid (4.7\%), different types of 
flavonoids. These components concluded that Cynaroside, Cosmosine, Ramno-citrine, Isoquercitrin, Ursolic acid $[6,7]$. The results showed that this compound have therapeutic effects such as Anti Flatulence, Mild Tranquilizer, Anti Spasm, Anti-Bacterial, Anti-Viral, Oxidative [2, 6]. This plant has free radical inhibitors that reduce the incidence of cancer, Parkinson's disease, Alzheimer's disease and atherosclerosis $[8,9]$. Studies in the UK showed that Melissa was Anti fatigue, sedative, Anti- anxiety and sleepy and feed intake of Melissa is reduced anxiety in females $[10,11,12,13]$ that these agents cause Acne.

Among useful herbals used for acne treatment, Lamiaceae family plays important roles in some therapies, because these plants contain Phenol and essential anti-bacterial oils, Poly-phenols, Terpen and Tannin [7].

Genuine Melissa oil is pale yellow sweet smelling oil with an odour of aromatic lemon. Yields are as low as $0.014 \%$ for distillation of the fresh herb to $0.112 \%$ for dried herb, increasing to $0.13 \%$ using cohobating. True oil is extremely expensive. The oil is held in high esteem for its use in aromatherapy, it is reported to have a sedative effect $[14,15]$. Also, the herb has potent uses in skin and body care. Lemon balm hydrosol is added to clay masks for skin healing [7].

Acne and infectious diseases of the skin are responsible for a significant part of the annual cost of health care [1]. These diseases, in addition to being chronic, impose a lot of psychological stress on the patient, which justifies the need for some patients to seek complementary and alternative therapies. The use of complementary and alternative medicine, including medicinal plants, is common among patients with acne and infectious skin diseases [16, 17]. Acne is an infectious and inflammatory disease and one of the most common human diseases [18]. In addition to anti-acne herbal remedies that have been traditionally used, several antibacterial activities of several plants have been investigated to evaluate their ability to treat acne. Plants that contain Silicic acid, sulfur components, Tannin and Anthranoids are used to treat acne. Some of these herbal medicines are tea tree extract, Aloe Vera, and Melissa officinalis, Elymus repens, Taraxacum officinalis, Equisetum arvense, Ferula sp., Allium and Brassicaceae family [1, 19, 20].

Due to the fact that there is no similar study on herbal therapists, its compatibility with other reports is not possible. Treatment should be continued for four to six months, and the team has shown that after 3 to 5 years after the treatment, the person remains free of acne, but usually once treatment is not adequate and recurrence is observed in some cases. It is advisable to adjust the dose and treatment period with each individual's response. Considering that in $70 \%$ of cases, this treatment will result in a complete recovery, and 30\% will either need longer treatment or do not respond at all, which can cause a lot of causes, including hormonal disorders, and as long as the problem arises, treatment of Iso-tretenoin is not sufficient [2].

It is traditionally used as a mild sedative, spasmolytic and antibacterial agent. In addition, results of a lot of clinical researches have shown that essential oil of lemon balm can be used in treatment of some disease, as antioxidant against negative effects of free radicals and it has a positive effect on immune system and stress. By means of its antimicrobial activity it was found to be effective in the perfume and cosmetic industry, due to hydrosol in its essential oil [21].

However, many studies have not done about the effect of lemon balm (Melissa officinalis) on acne but regarding the effects of her sterilization on herpes, the results show the positive effects of herbal application. So that Melissa extract formulated as creams in a double-blind, Placebocontrolled, randomized trial in 66 patients with recurrent herpes lips were investigated. The cream was applied four times a day for 5 days to the affected area. Treatment with Melissa significantly increased the healing rate compared with the placebo, prevented the infection from spreading, and soothed the blister and pain [22]. Then this plant used for treatment of stress insomnias, hysterical nervous stimulations, Melancholy, treatment of gastro-duodenal ulcers, nervous weakness, gastrointestinal diseases. It is used as a mouth wash for management of oral cavity ulcers, Because of comprising vitamin C, application topically for Herpes is beneficial. Its oil is used for massages, after shower lotions and pain reliefs caused by rheumatic diseases and neck stiffness as well.

Acne one of the skin problems that almost everyone's involved is acne which is caused by different reasons such as stress, hormonal imbalances like Pre Menstrual Syndrome (PMS), skin 
contamination, bacterial activities of Propiony bacterium acnes, drugs, digestive system like liver disease, defecation problems or internal organs inflammation, air and environmental pollution [23].

As regards many plants have inhibitory effects on the growth of bacteria, fungi and viruses in external conditions. Also, some plants have anti-inflammatory and anti-inflammatory properties. However, there is little clinical evidence about the efficacy and safety of these plants in the treatment of acne and other skin infections.

\section{Materials and Methods}

Therefore, we choose this specious for investigation of the topical effects of lemon balm on Acne. For this purpose, was used organic and domesticated plant of research farm of Ferdowsi University of Mashhad. In Iranian complementary and alternative medicine were used some part of Melisa for some therapy. Then be use stew of aerial organs (shoot and leaves) before flowering, fresh or dried up hydro alcoholic extract, capsules of dry plant powder are advantageous. But in this experiment we use extract of leaves and aerial part of Melissa.

This experiment was based on a randomized block design with 3 replications. Two factors were 2 levels of treatment extract (hydro distillation extract and hydro alcoholic extract of Melissa) and 3 levels of age's group. For this study we chose 21 people in two groups that they had 15-35 years old. The groups concluded 21 people in 4 sub group that separated in (15-21), (22-28) and (29-35) years old and 3 control candidate. The control group cleaned the face by shampoo (pH:7) only. We have 3 people per group. Acne was arising from stress, skin pollution by cosmetics, job types and change level of hormones from stress condition in all clients.

In both of two groups, all lab test to be done to sure all clients didn't have any hormonal and bad effect of gastronomical disease that research condition was controlled.

The procedures have been done in adherence to the Declaration of Helsinki of 1975 revised in 2013.

In this study, two protocols were followed and applied on the clients.

\section{The first protocol (water extract)}

In this protocol all people in the first group, were used to water extract the active ingredient: for this kind of essential oil extract we used 30 gr. of dried shoot and leaves parts of Melissa and added $500 \mathrm{ml}$. distilled water in 4 hours and set by Clevenger system [24, 25].

The clients were requested to follow this program. At first, they must wash the skin for 3 months with baby shampoo (ph.7) then they should be sprayed hydro distillation extract 3-5 times daily.

\section{The second protocol (Hydro alcoholic extract)}

This group used Hydro alcoholic extract solution. To prepare this solution, $150 \mathrm{~g}$ dry and chopped leaves were used. The amount of herbal substance was soaked in $100 \mathrm{ml}$. of $25 \%$ ethyl alcohol for 10 days and kept in dark container in room temperature $\left(21^{\circ} \mathrm{C}\right)$. After 10 days, the mixture was straightened. Dissolve the tincture with 4 volumes of distilled water [24].

The second group must clean the skin same that first group but they should spray Hydro alcoholic extract 3 to 5 times daily on the clean skin.

To make the test more uniform, all clients followed a healthy diet and fit each person's condition.

The results were analyzed by MSTAT-C and performed to Duncan's multiple range test on $(\alpha=0.05)$ probability and graphs draw in Excel software.

\section{Results and Discussion}

These results are documented by viewing and asking questions from clients and viewing skin changes. The results showed that the effect of hydro distillation and hydro alcoholic extract on 2 group of clients were significant ( $p>95 \%)$. 
Percent of affection after 3 months is shown on Fig. 1. These results indicated a positive effect of hydro distillation extract on Acne and Pimple treatment of 65\%. Also, the skin pores in $75 \%$ of clients were recovered after using this extract. This extract had positive effect to improve the secretion of the sebaceous glands on the client's skin. So that more than $92 \%$ of clients had improved after the end of the trial period.

In another group, we obtained the same result. Results for the controlled effect of hydro alcoholic extract on the client skin after 3 months showed that the skin pores treated in $75 \%$ of clients (Figure1). Acne and pimple in $95 \%$ of clients were prepared and $90 \%$ of them improved the sebaceous gland secretion as well as.

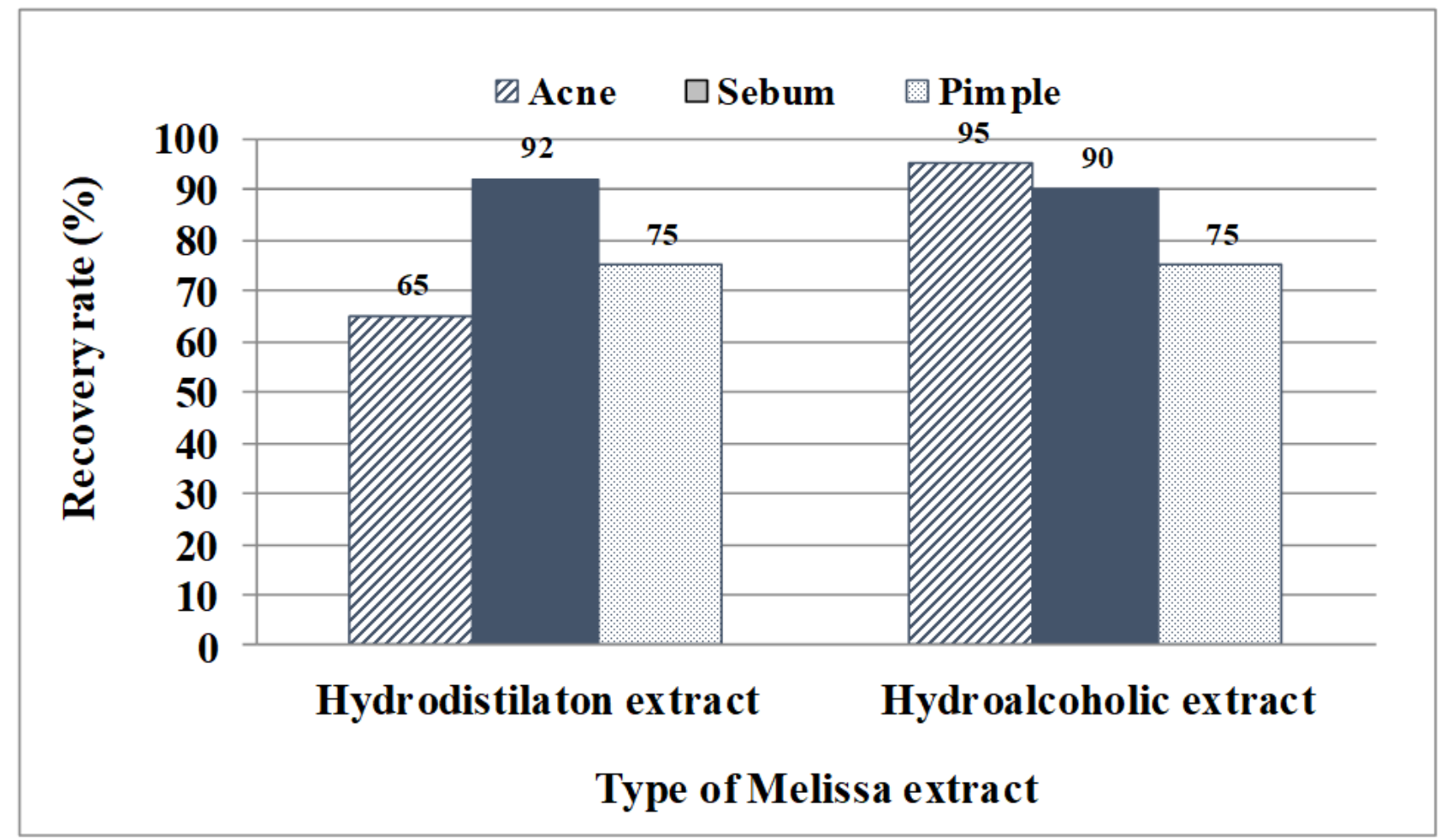

Figure 1. Recovery rate of Acne, Sebum and Pimple clients after using different Melissa extracts.

The studies indicated a reduction in stress and hormonal balance in the volunteers, which itself improves acne, and probably due to the aromatherapy effects of lemon balm, whose synergistic effect on acne treatment should be considered. Comparative effect on clients obtained that the best result was after hydro alcoholic extract treatments. The therapeutic effect of Hydro alcoholic extract on patients was significantly more than the hydro distillation extract. Also, the effect of Hydro alcoholic extract application on acne treatment was more than sebum and pimple treatment.

Skin changes before treatment (a) and after treatment (b) are observed in Picture 1 and 2. 


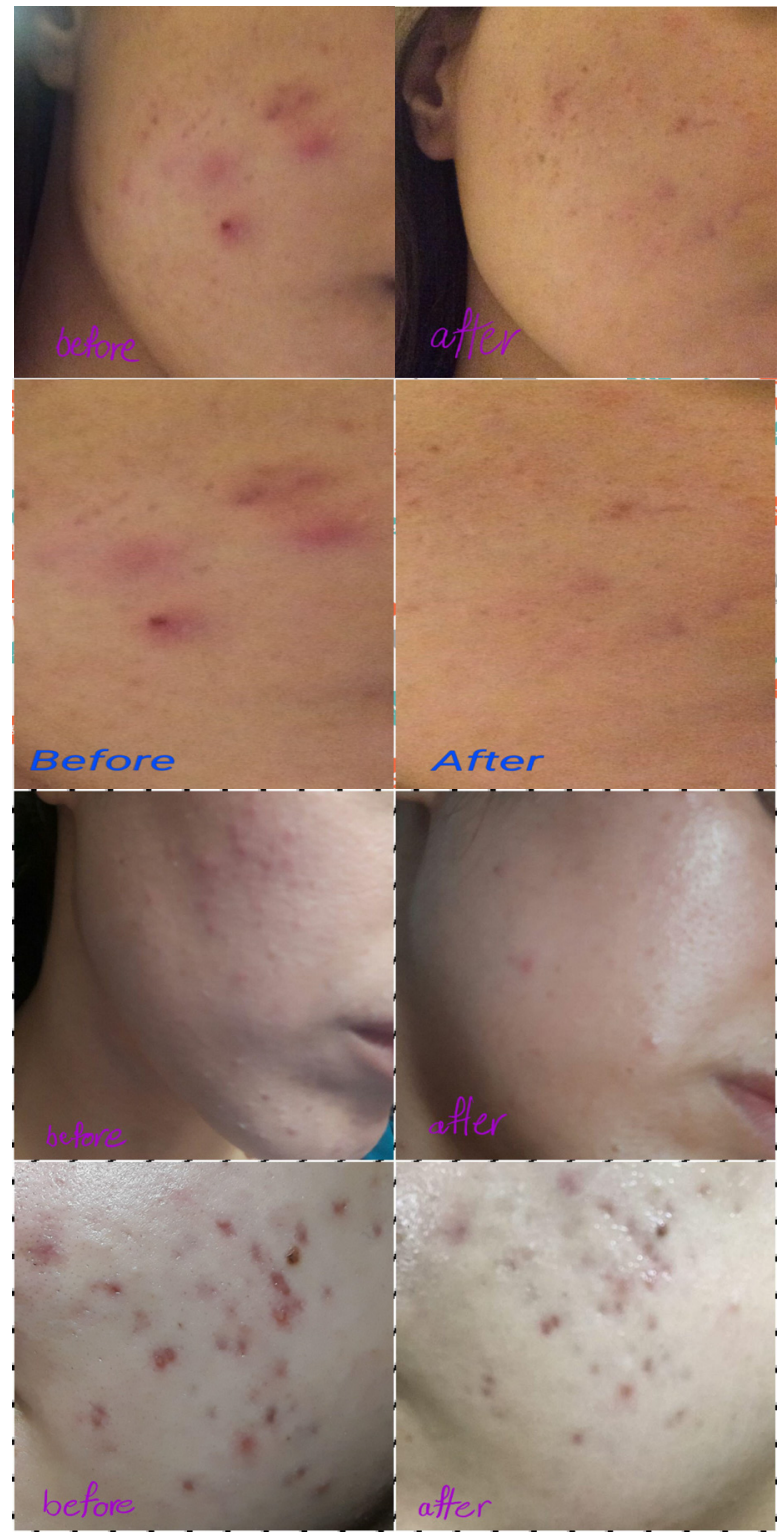

a

b

Picture 1. Skin changes before (a) and after (b) treatment by hydro alcoholic extract.

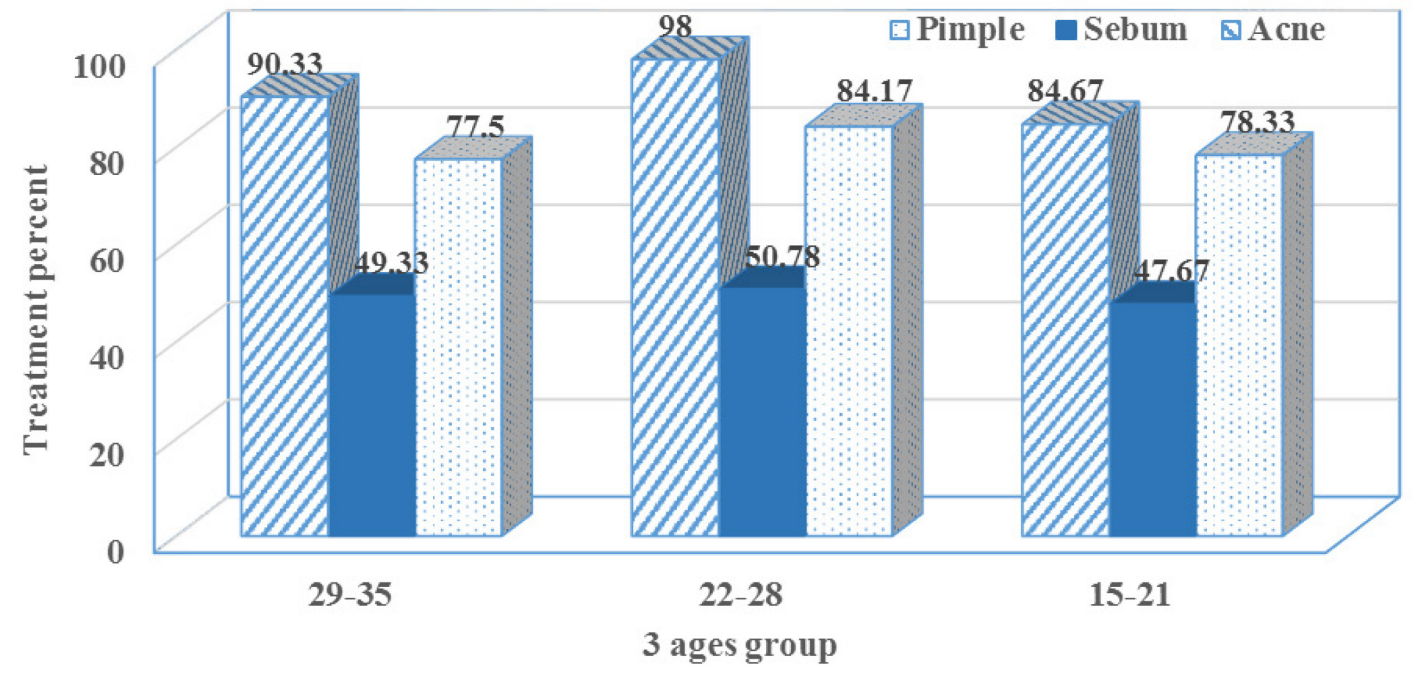

Figure 2. Interaction effect of age's group and 2 treatment liquid on Pimple, Acne and Sebum. 


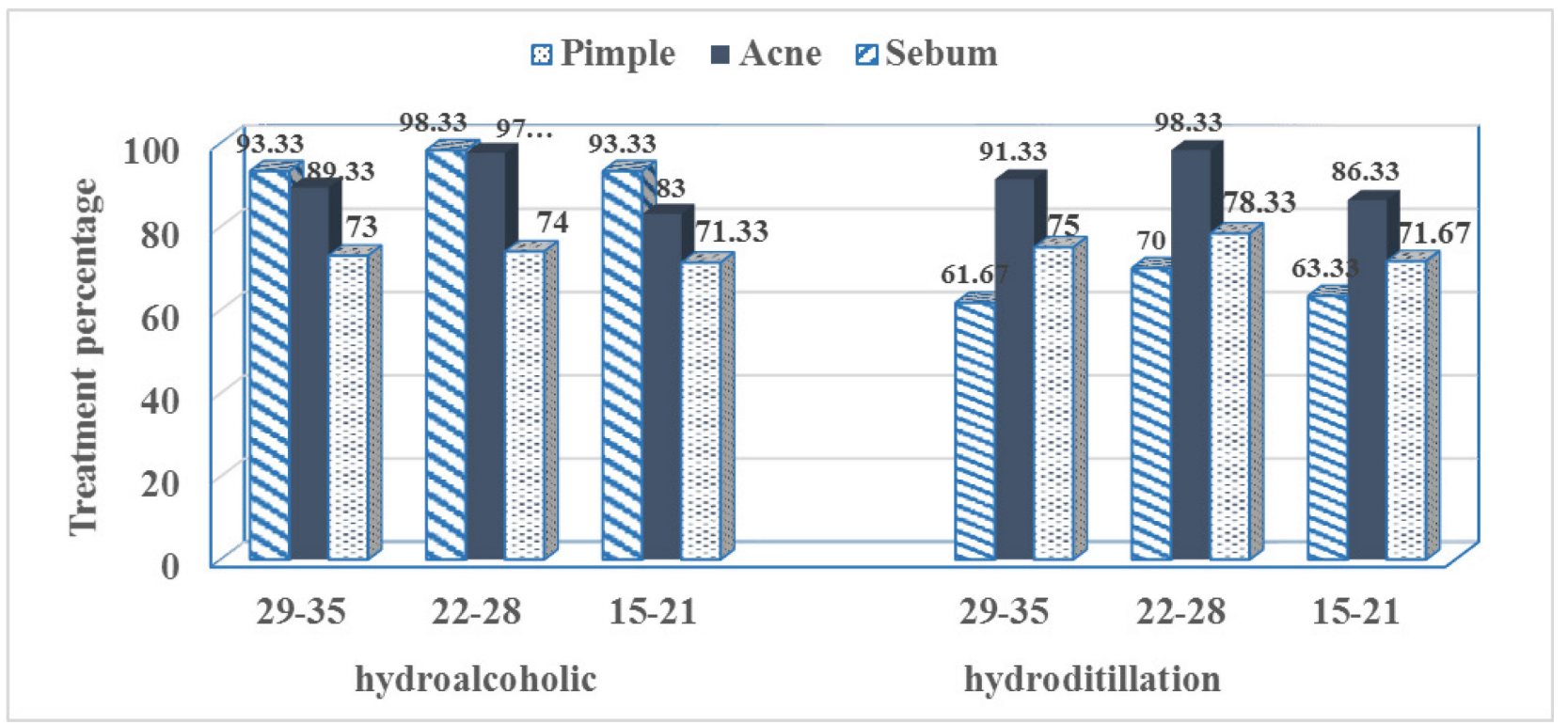

Figure 3. The interaction effect of hydro distillation extract treatment and client ages on treated the Pimple, Sebum and Acne.

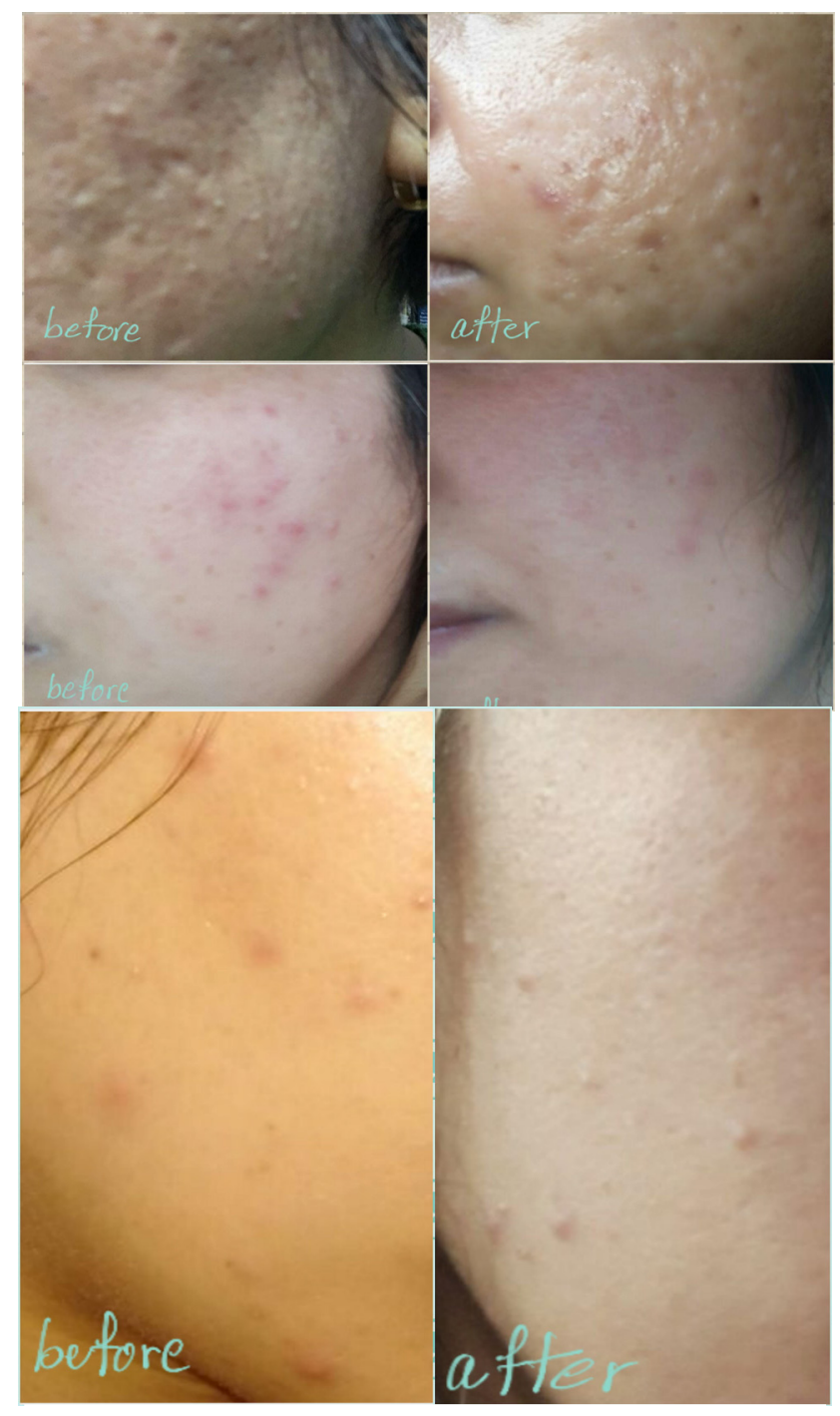

a

b

Picture 2. Skin changes before (a) and after (b) treatment by hydro distillation extract 
Plants with antimicrobial effects with different and even different microorganisms from antibiotics inhibit the growth of bacteria and this necessitates more comprehensive research in the field of medicinal plants [26]. This justifies the increasing increase in published articles on the antimicrobial properties of plants [27].

\section{Conclusions}

Intake antibiotic or topical lotion is one of the common ways to treat Acne. Unfortunately, in recent years there have been reports of bacterial resistance especially propenobacteria to antibiotics that justify the use of non-antibiotic treatments [28, 8]. Some foods cause acne, which is more likely to come from sweets and dairy products with high levels of glycaemia. Therefore, nutrition with healthy plants and low calorie foods, especially in diabetic patients, reduces the incidence of acne [29]. Therefore, to make the test more uniform, all clients followed a healthy diet and fit each people condition. The result showed that the positive effect of Melissa extract on acne treatment.

Considering that the treatment with both hydro alcoholic extract and aqueous extract on acne, pimple and sebum treatment had a positive and decreasing effect, and given the negative effects of the use of chemical and antibacterial drugs and after confirmation of definite doses of herbal therapists such as Melissa extract, these compounds are to be used. It should be noted that a skin scan will be used to determine the exact amount of treatment for new ones. In the follow-up, the skin changes were recorded using a scan device.

It seems to the best results to observe in (22-28 year) ages group after use of hydro alcoholic extract of Melisa that is same in Acne treatment in this age's group after use of hydro distillation extract. The best result was obtained in the 14-20 years old group. The result showed that the positive effect of therapy in 29-35 years old group was longer than other groups. These results are due to the sustained long-term effects of the disease and the adverse effects of diets and dietary and psychological behaviours in this group. It seems that the presence of alcohol in the hydro alcoholic extract increases the skin's disinfection properties, and therefore the effect of this type of extract may be due to the increasing effects of alcohol.

There is not scientific study about the investigation of Melissa extracts on skin care the except that the Melissa ointment had positive effect on shortening of the healing period, the prevention of a spreading of the infection and the rapid effect on typical symptoms of herpes like itching, tingling, burning, stabbing, swelling, tautness and erythema [30]. The different mechanism of action of the balm mint extract rules out the development of resistance of the herpes virus. Some indication exists that the intervals between the periods with herpes might be prolonged with balm mint cream treatment, but reports indicate that the use of ointments, lotions, creams or cosmetics containing Melissa extract has had a positive effect on the skin, because the hydrosols can be used externally in skin care products in the form of a shower or diluted into a tonic drink [31].

Today, lemon balm is used in various branches of industry (such as medicine, perfume and cosmetic, and food etc.) in a lot of countries of the world [30], so the scientist must be research about the different therapy effect of Melissa on health.

\section{Conflict of Interest}

The author declares that there is no conflict of interest.

\section{Acknowledgements}

Many thanks of all clients who let us published the pictures and result of treatment. 


\section{References}

[1] Anonymous, Minor oil crops - Individual monographs (Melissa officinalis-Milfoil-NindePatchouli-Tarragon).

[2] P. Sandra, C. Bicchi, Capillary Gas Chromatography in Essential Oil AnalysisChromatographic Methods, Heidelberg: Huethig, 1987

[3] R. Awad et al., Bioassay-guided ractionation of lemon balm (Melissa officinalis L) using an in vitro measure of GABA transaminase activity, Phytotherapy Research. 23(8) (2009)10751081.

[4] S. Arctander, Perfume and Flavour Materials of Natural Origin. Lulu.com, 2017. ISBN 0244329214.

[5] M.R. Nilforoushzadeh, Information on: https://paperpdf.ir/10007339, (2017).

[6] S. Esmaeilzadeh Bahabadi, A. Rezaei, Nitric Oxide Increased the Rosmarinic Acid and Essential Oil Production in in-vitro-cultured Melissa officinalis, Journal of Medicinal Plants 17(65) (2018) 61-72.

[7] S. Kyanbakht, Pharmacological studies in acne plant treatment and infectious diseases: a systematic review, Medicinal Plants Quarterly Journal. 4(48) (2013) 13-25.

[8] Anonymous (SAEOPA and KARWYL Consultancy), Essential Oil Crops, Production guidelines for lemon balm, Lemon balm production, Pub. Directorate Communication Services Department of Agriculture, Forestry and Fisheries. (2016 ), pp. 1-16.

[9] A. Kamalifar, Study of the therapeutic effect of mint sweat on acne, a clinical trial, National Conference on Medicinal Plants, (2010). Available: https://www.civilica.com/PaperHERBAL01-HERBAL01_1237.html.

[10] D.O. Kennedy et al., Anxiolytic effects of a combination of Melissa officinalis and Valeriana officinalis during laboratory induced stress, Journal of Psychopharmacology. 20(2) (2004) 96102.

[11] A.E. Taiwo et al. Anxiolytic and antidepressantlike effects of Melissa officinalis (lemon balm) extract in rats: Influence of administration and gender, Indian Journal of Pharmacology. 44(2) (2012) 189.

[12] N.H. Ismail et al., High glycemic load diet, milk and ice cream consumption are related to acne vulgaris in Malaysian Young adult, BC Dermatol. 16 (2012) 12-13.

[13] Anonymous, Lemon Balm/Melissa Benefits \& Information (Melissa Officinalis). Available: https://www.herbwisdom.com/herb-lemon-balm.html, (2018), 1-5.

[14] B.S.W. Dawson, R.A. Franich, R. Medler, Essential oil of Melissa officinalis 1. subsp altissima. (Sibthr. et Smith), Flavour and Fragrance Journal. (1988) 167-170.

[15] B.M. Lawrence, Progress in Essential Oils, Perfumer and Flavorist. 8(2) (1983) 61.

[16] N. Smith et al., Use of complementary and alternative medicine among adults with skin disease: results from a national survey, J. Am. Acad. Dermatol. 60 (2009) 419-425.

[17] Ernst E and Fugh-Berman A. Complementary and alternative medicine: what is it all about? Occup. Environ. Med. 59 (2002) 140-144.

[18] P.J. Magin et al., Topical and oral CAM in acne: a review of the empirical evidence and a consideration of its context, Complement. Ther. Med. 14 (2006) 62-76.

[19] Kraft K. Erkrankungen der haut (II). Weitere ekzemformen, akne und pruritus.

[20] Diseases of the skin (II). Other eczema types, acne and pruritus, Z. Phytotherapie. 28 (2007) 129-133. 
[21] R. Bahtiyarca Bağdat, B. Coşge. The essential oil of lemon balm (Melissa officinalis L.), its components and using fields, J. Fac. Agric. OMU. 21(1) (2006) 116-121.

[22] Z. Jelodarian et al., Plant Sulfides: Effective Compounds on Hair and Skin Diseases, Journal of Isfahan Medical School. 30(217) (2013) 2198-2205.

[23] A. Nast, B. Dreno, European evidence-based (S3) guidelines for the treatment of acne. Journal of the European Academy of Dermatology and Venereology. 26 (2012) 1-29.

[24] S. Khosroshahi et al., A Comprehensive and photographic Guide to Herbal Pharmacy. Sabziran Agriculture Institute, Second edition, 2011, pp. 79-85.

[25] M. Ghayour et al., Investigating the Anti-epileptic and Sedative Effects of Hydroalcoholic Extract of Melissa officinalis (Lemon Balm) Leaf on Pentylenetetrazol Induced Epileptiform Seizures in Wistar Rat. JMP 1(41) (2012) 64-73.

[26] F.A.E. Gharib, Effect of salicylic acid on the growth, metabolic activities and oil content of basil and marjoram, Int. J. Agri. Biol. 4 (2006) 485-492.

[27] S.E. Wolverto, J.C. Harper, J. Am, Important controversies associated with isotretinoin therapy for acne, Clin Dermatol. 14(2) (2013) 71-76.

[28] J.P. Kamdem, A. Adeniran, A.A. Boligon, Antioxidant activity, genotoxicity and cytotoxicity evaluation of lemon balm (Melissa officinalis L.) ethanolic extract: Its potential role in neuroprotection, Ind. Crops Prod. 51 (2013) 26-34.

[29] M. Bayat et al., Neuroprotective properties of Melissa officinalis after hypoxic-ischemic injury both in vitro and in vivo. DARU. 20 (2012) 42.

[30] R. Koytchev, R.G. Alken, S. Dundarov, Balm mint extract (Lo-701) for topical treatment of recurring herpes labialis, Phytomedicine. 6 (1999) 225-230.

[31] J. Rose, An Herb for Skin Care, Aromatic Plant Project Pres Release. 8 (2000) 897-901. 